\title{
Wrist Joint Flexor Tendons
}

National Cancer Institute

\section{Source}

National Cancer Institute. Wrist Joint Flexor Tendons. NCI Thesaurus. Code C161385.

The tendons located along the inside of the forearm that cross at the wrist and connect muscles of the forearm to wrist and hand bones, enabling bending of the wrist towards the palm. 